\title{
Finesse Limits in Hollow Core Fiber based Fabry-Perot interferometers
}

\author{
Meng Ding, Eric R. Numkam Fokoua, Thomas D. Bradley, Francesco Poletti, David J. Richardson, \\ Fellow, IEEE, Fellow OSA, and Radan Slavík, Senior Member, IEEE, Fellow OSA
}

\begin{abstract}
Due to their low sensitivity to changes to the external environment, low optical nonlinearity, low chromatic dispersion, and compatibility with fiber systems, hollow-core optical fibers (HCFs) represent an ideal medium for fiber Fabry-Perot interferometers (FPs). Many applications can benefit from the availability of FPs with high finesse or high finesse-length product. However, the mechanisms that limit the performance of HCFbased FPs are yet to be fully elucidated to the best of our knowledge. In this paper, we present a comprehensive analysis of several factors that impact HCF-FP performance and limit their finesse, e. g., mirror tilt, the distance between the HCF end-face and mirror, $\mathrm{HCF}$ cleave angle and $\mathrm{HCF}$ attenuation. In a sequence of experiments, we built and characterized five HCF-FPs with lengths ranging from $0.65 \mathrm{~m}$ to $9.25 \mathrm{~m}$. By fitting the experimental data with derived analytical expressions, we found the mirrorassisted coupling loss to be below $-0.0028 \mathrm{~dB}$ (corresponding to a coupling efficiency of $\mathbf{9 9 . 9 4 \%}$ ), which should allow finesse values greater than 5000 to be achieved. Experimentally, we demonstrate here a value of 2400 , limited by the parameters of the mirrors available to us presently. We then show that the low coupling loss and high repeatability of mirror alignment and HCF cleave quality allows the effective use of such high-finesse FP for reliable measurements of $\mathrm{HCF}$ attenuation, even with short fiber length samples (10 $\mathrm{m}$ in our demonstration).
\end{abstract}

Index Terms-Fabry-Perot, interferometers, hollow-core fibers, optical fiber devices

\section{INTRODUCTION}

$\mathrm{F}$ ABRY-PEROT interferometers (FPs) with high finesse, typically formed by enclosing an optical path with two highly reflective mirrors, present many unique properties such as high wavelength selectivity, high extinction ratio, and the ability to enhance light intensity inside the resonant cavity, making them a common device in many applications such as lasers [1], metrology [2], [3], and sensing [4]-[6].

Besides high finesse, the performance in many applications is improved when the FP cavity length is increased. For example, in lasers, longer cavities often enable higher output powers to be achieved. Another example is metrology in which a longer cavity produces narrower transmission peaks, improving the accuracy of many measurements. However, longer cavities are more prone to misalignment (and require

Manuscirpt received December xx, xxxx; revised February xx,xxxx; accepted $\mathrm{xx}, \mathrm{xxxx}$. This work was supported by EPSRC project "Airguide Photonics", under grant EP/P030181/1. This work of Meng Ding was supported by CSC scholarship. Francesco Poletti gratefully acknowledges EU ERC (grant 682724); Radan Slavík and Eric R. Numkam Fokoua were supported by RAEng Fellowship. more accurate alignment in the first place) and their length is often limited by the available physical space. These shortcomings can be addressed with the use of a hollow-core fiber (HCF). HCF can be coiled and the mirrors need to be only aligned with the fiber input and output (rather than aligning two distant mirrors with respect to each other as in the case for a free-space FP). Furthermore, the hollow regions in a HCF can be filled with materials of interest, e.g., a gas to act as a laser gain medium [7]-[10], gases/liquids for customized nonlinear interactions [11]-[13], or analytes in sensing applications (e.g., in cavity ring-down spectroscopy [14]).

HCF can also be advantageous for applications that do not require access to the light path and for which standard singlemode fiber-based FP (SSMF-FP) cavities might be considered. In SSMF, light travels through silica glass which has relatively large thermo-optic coefficients, causing the optical length of the SSMF-FP (and thus also spectral position of its transmission peaks) to change significantly with temperature. This unwanted sensitivity was demonstrated to be $\sim 15 \mathrm{x}$ smaller in a HCFbased FP (HCF-FP) than in a SSMF-FP [15], [16] and can be further reduced, or even made zero, at low temperatures [17]. Another advantage of HCF-FP over SSMF-FP is its three orders of magnitude lower non-linearity (when HCF is filled with atmospheric air [1] or even lower when evacuated [18]), allowing significantly higher optical powers to be used to probe it. Finally, SSMF loss and dispersion outside of the communication wavelength band can also pose limitations. Recent improvements in the fabrication of HCFs have resulted in losses as low as $0.28 \mathrm{~dB} / \mathrm{km}$ in the C-band, a mere factor of 2 above that of the SSMF $(0.28 \mathrm{~dB} / \mathrm{km}$ in HCF [19] vs 0.14 $\mathrm{dB} / \mathrm{km}$ for SSMF [20]). At both shorter and longer wavelengths, the latest generation of HCFs outperform solid glass fibers in terms of loss [21], [22], [24], [25] whilst having chromatic dispersion of only a few $\mathrm{ps} / \mathrm{nm} / \mathrm{km}$ over several hundred nm's of optical bandwidth [16]. Most of these results were achieved with the Nested Antiresonant Nodeless Fiber (NANF) geometry [23]. Such low-loss values would enable high finesse HCF-FPs for multitude of applications at any wavelength of choice.

There have been several reports of high-finesse (up to 160) HCF-FPs. The cavity mirrors in those works were formed either

Meng Ding, Eric R. Numkam Fokoua, Thomas D. Bradley, Francesco Poletti, David J. Richardson, and Radan Slavík are with the Optoelectronic Research Centre, University of Southampton, SO17 1BJ, UK (M.Ding@soton.ac.uk; R.Slavik@ soton.ac.uk). 
by using photonics crystal slabs [24], or high reflectivity dielectric coatings [16], [25]. The highest finesse-length product (that determines the transmission peak width) was achieved in a 22-m long all-fiber FP cavity with a finesse of over 120 [16], achieving $47 \mathrm{kHz} 3-\mathrm{dB}$ transmission peak width.

Many applications would benefit from higher finesse or finesse-length product. For example, in cavity ring-down spectroscopy, the sensitivity is proportional to the finesse [14], [26]. In cavity-enhanced photothermal FP gas cell sensors, high finesse improves the photothermal phase modulation as well as the sensitivity of phase detection [27]. When FPs are used as filters, the extinction ratio improves with finesse [28]. When used as a frequency reference for laser locking [3] or a frequency discrimination element for strain sensing [6], the frequency discrimination capacity improves with finesse-length product.

To achieve higher finesse than reported so far, the net reflectivity (amount of light that is back-reflected from the mirror and back-coupled into the HCF) needs to be increased. For longer cavities, the propagation loss in HCF is also likely to limit the achievable finesse or finesse-length product. These limitations have not, to the best of our knowledge, been studied yet.

In this paper, we explore the performance limits of HCF-FPs in detail, following our recent preliminary study [29]. We demonstrate that the coupling loss between the HCF and the external mirrors (mostly limited by the HCF end facet quality) can be as low as $0.0028 \mathrm{~dB}$. This allows a finesse of over 5000 to be achieved when mirrors with optimized reflectivity are used. Experimentally, we achieved a finesse of 2400 , limited by the reflectivity of the available mirrors $(99.8 \%)$, which is only by a factor of two lower than the above-mentioned limit of 5000 . Nevertheless, the achieved value is over an order of magnitude higher than demonstrated previously. Considering the state-ofthe art HCF loss (NANF geometry, $0.28 \mathrm{~dB} / \mathrm{km}$ [19]) and the mirror coupling loss reported herein, the largest finesse-length product achievable is $47 \mathrm{~km}$, producing transmission peaks as narrow as $3.2 \mathrm{kHz}$. Experimentally, we demonstrate $13 \mathrm{kHz}$ in an $\mathrm{HCF}$ with a loss of $0.65 \mathrm{~dB} / \mathrm{km}$, which is four times lower than demonstrated previously in a HCF-FP [16].

Besides studying the HCF-FP performance limits, we also demonstrate how a HCF-FP can be used to accurately characterize the loss of a HCFs using a short piece of the fiber (e.g., in our demonstration, a loss of $0.65 \mathrm{~dB} / \mathrm{km}$ is measured using only a $10 \mathrm{~m}$ HCF sample).

\section{HCF-FP STRUCTURE AND ITS ANALYSIS}

\section{A. HCF-FP set up}

The structure of our HCF-FP, including the light coupling arrangement is shown in Fig. 1. The HCF is a NANF, the crosssection of which is shown as inset in Fig. 1. In experiments, we used the NANF sample used in [30] with a transmission loss measured using the cut-back method of $0.65 \mathrm{~dB} / \mathrm{km}$ [30]. The NANF sample was first cleaved at both ends with the end-face quality carefully inspected for any cracks or irregularities. Both fiber ends were put onto 3-axes micro-positioning stages. The transmissivity of the two cavity mirrors (2-mm thick silica glass with antireflective coating on one side and high-reflective thin film dielectric coatings on the other side, reflectivity specified by the manufacturer, Union Optics, China as " $>99.8 \%$ ") was measured to be $-32.6 \mathrm{~dB}(0.055 \%)$ and $-31.1 \mathrm{~dB}(0.078 \%)$. From these measurements, the maximum possible reflectivity (that could be achieved only if the mirrors have no internal loss) was calculated to be $99.945 \%$ and $99.922 \%$ respectively. The two mirrors were mounted on tilt and yaw mounts and put close to the HCF end faces.

Light was launched into the FP from SSMF using a pair of lenses with their focal length ratio chosen for efficient coupling from SSMF (mode field diameter (MFD) of $10.4 \mu \mathrm{m}$ ) to NANF (MFD of $24 \mu \mathrm{m}$ ). At the FP output, identical optics was used to couple light back into the SSMF.

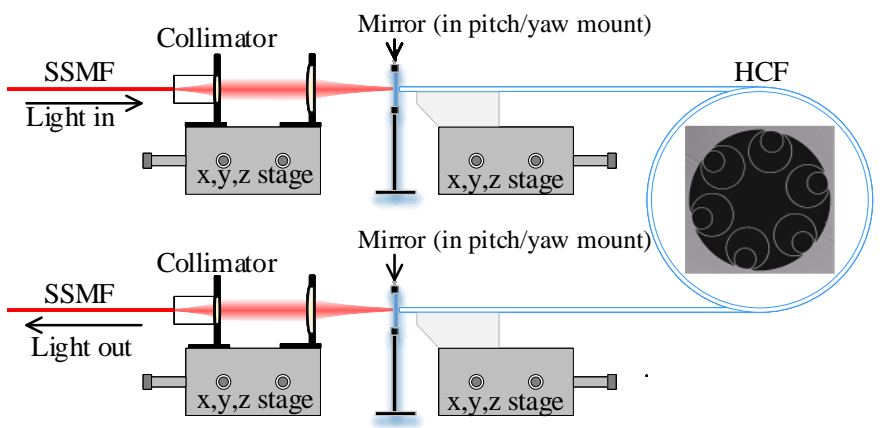

Fig. 1 HCF-FP with light coupling to and from an SSMF. Inset: a crosssectional scanning electron microscope (SEM) image of the HCF microstructure.

\section{B. Key FP parameters}

Before presenting results, let us introduce the key FP parameters. A FP's transmission spectrum can be given by [24]

$$
T(v)=\frac{t_{\text {resonance }}}{1+\left(\frac{2 F}{\pi}\right)^{2} \sin ^{2}\left(\frac{\pi v}{F S R}\right)},
$$

where $v$ is the optical frequency. The finesse $(F)$, transmission at the resonance $\left(t_{\text {resonance }}\right)$ and free spectral range (FSR) are defined below. $F$ is given by [31]:

$$
F=\frac{\pi \sqrt{R \cdot A}}{1-R \cdot A}
$$

where $R$ is the effective reflectivity of the mirrors. For an HCF$\mathrm{FP}$, it depends on the two mirror reflectivities $r_{1,2}$ and the coupling efficiencies $\eta_{1,2}$ between the HCF and the mirrors, so $R=\sqrt{\eta_{1} r_{1} \eta_{2} r_{2}}$. In other words, $\mathrm{R}$ characterizes how much light at the two fiber outputs is coupled back into them. $A=$ $10^{\left(-\alpha_{l} L / 10\right)}$ is the light power loss in HCF in a single pass with $\alpha_{l}$ being the $\mathrm{HCF}$ attenuation in $\mathrm{dB} / \mathrm{m}$, and $L$ the HCF length used in the FP.

FSR is defined as the spectral distance between the transmission peaks, which for fiber-FP is given by: 


$$
F S R=\frac{c}{2 n_{g} L},
$$

where $c$ is the speed of light in vacuum, $n_{\mathrm{g}}$ is the HCF group refractive index, and $L$ the fiber length.

Regarding the transmission at resonance, $t_{\text {resonance, }}$ it is typically expressed in $\mathrm{dB}$ as insertion loss (IL):

$$
I L=10 \cdot \log _{10}\left[\frac{t_{1} t_{2} A}{(1-R A)^{2}}\right] .
$$

Here, $t_{1,2}$ is the mirror transmissivity (which we mentioned earlier to be $0.055 \%$ and $0.078 \%$ for our mirrors). Considering symmetric FP structure (both FP reflections identical), IL rises due to the loss in HCF (via $A$ ), limited coupling between HCF and mirror (via $\eta_{1,2}$ ), and loss in the mirrors (via $r_{1,2}+t_{1,2}<1$ ).

Another FP parameter, which is of interest in many applications, is the full width at half of maximum (FWHM) of the transmission peaks:

$$
\Delta v=\frac{F S R}{F} .
$$

Substituting FSR using Eq. (3) we found that it depends inversely on the length-finesse $(F L)$ product.

The Resonator quality factor $\mathrm{Q}$ is then defined as:

$$
Q=\frac{v}{\Delta v}
$$

\section{Coupling efficiency $\eta_{1,2}$}

There are several factors that influence the coupling efficiency, i.e., fiber-mirror gap, mirror-tilt and cleave quality (angle and overall flatness) of the HCF. The coupling efficiency is given by:

$$
\eta=\frac{\left|\iint E_{r} E_{0}^{*} r d r d \theta\right|^{2}}{\iint\left|E_{r}\right|^{2} r d r d \theta \iint\left|E_{0}\right|^{2} r d r d \theta},
$$

where $E_{0}$ is the transverse electric field at the $\mathrm{HCF}$ end facet and $E_{\mathrm{r}}$ is the reflected field when $E_{0}$ emerges from the $\mathrm{HCF}$, is reflected from the mirror, and reaches the HCF end facet. Further, we refer to $\eta$ also as the 'coupling loss', which is the coupling efficiency $\eta$ expressed in dB.

For illustrative purposes, we calculate how the HCF-FP performance changes due to mirror tilt, its distance from the $\mathrm{HCF}, \mathrm{HCF}$ cleave angle, and HCF loss (Fig. 2-Fig. 4) using the above equations. For this calculation we assume that the HCF fundamental mode has a Gaussian shape with a MFD = $24 \mu \mathrm{m}$, and resonator mirrors with a reflectivity of $99.9 \%$ and transmission of $0.1 \%$ (loss-less mirrors). We set the integration limits to \pm 2 times MFD.

Fig. 2 shows the predicted FP performance and its degradation due to tilts in both mirrors and due to placing the mirrors at a finite distance from the HCF end facet. We see that placing mirrors more than $2 \mu \mathrm{m}$ from the HCF end facet brings significant degradation to both $F$ and $I L$. Mirror tilts are also very critical, especially when mirrors are less than $2 \mu \mathrm{m}$ from the HCF end facet, where tilt as small as $0.02^{\circ}$ produces appreciable change in both $F$ and $I L$. For a $2 \mu \mathrm{m}$ distance, to keep the $I L<-3 \mathrm{~dB}$, the tilt angle needs to be controlled to within $0.02^{\circ}$.

The minimum distance at which we can place the mirror from the HCF depends on the quality (flatness, angle) of the HCF cleave. Considering HCF with an outer diameter of $200 \mu \mathrm{m}$, we can calculate the minimum achievable distance between the HCF core center and the mirror for a given cleave angle. Although this may not be the dominant coupling loss factor related to the cleave quality, it is the most straightforward to evaluate theoretically and measure experimentally and can thus provide useful insight. As follows from Fig. 3 (a), the cleave angle should be below $0.5^{\circ}$ to avoid any appreciable degradation in $F$ or $I L$. Fortunately, this can be achieved with careful fiber cleaving using commercially-available fiber cleavers.

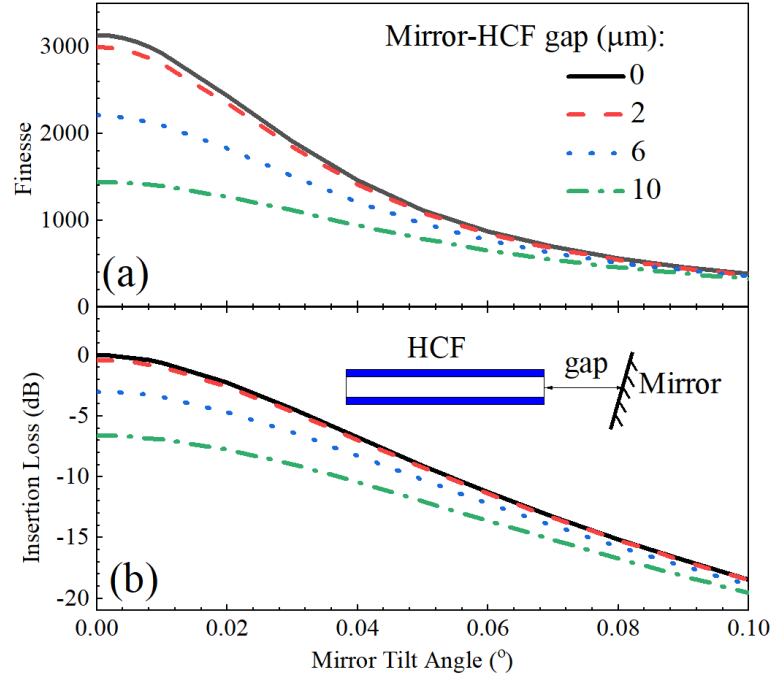

Fig. 2 (a) Finesse and (b) insertion loss versus mirror tilt angle, for different values of gap between the HCF end-facet and the mirror (black solid, $0 \mu \mathrm{m}$; red dash, $2 \mu \mathrm{m}$; blue dot, $6 \mu \mathrm{m}$; green dash dot, $10 \mu \mathrm{m}$ ).

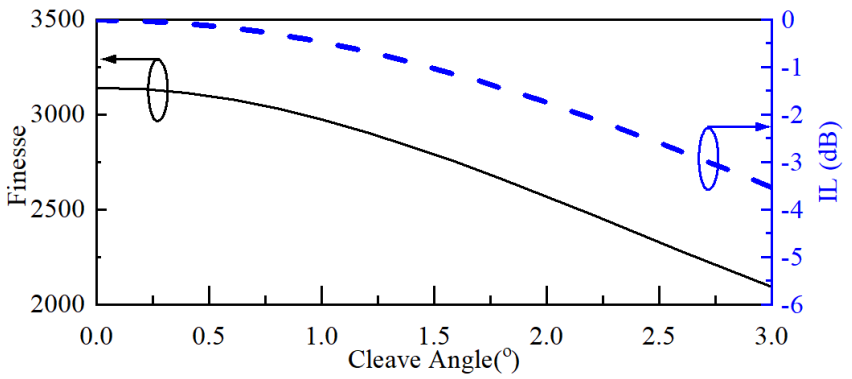

Fig. 3 Finesse (black solid) and insertion loss (blue dash) versus cleave angle of the HCF ends.

\section{Fiber attenuation}

Fig. 4. shows how $F, I L, \Delta v$, and $Q$ are influenced by HCF attenuation, considering the latest three generations of NANF with loss of $1.3 \mathrm{~dB} / \mathrm{km}, 0.65 \mathrm{~dB} / \mathrm{km}$ and $0.28 \mathrm{~dB} / \mathrm{km}$ [19], [30], 
[32] and the same FP parameters. As expected, both $F$ and $I L$ deteriorate with HCF length and attenuation. To keep the $I L<-$ $3 \mathrm{~dB}$, the HCF length needs to be limited to $2.5 \mathrm{~m}$ for HCF with an attenuation of $0.65 \mathrm{~dB} / \mathrm{km}$ and $5.7 \mathrm{~m}$ for HCF with attenuation of $0.28 \mathrm{~dB} / \mathrm{km}$. Values of $\Delta v$, and $Q$ keep improving even beyond these HCF lengths, reaching values in the range of $10 \mathrm{kHz}(\Delta v)$ and $10^{10}(Q)$.

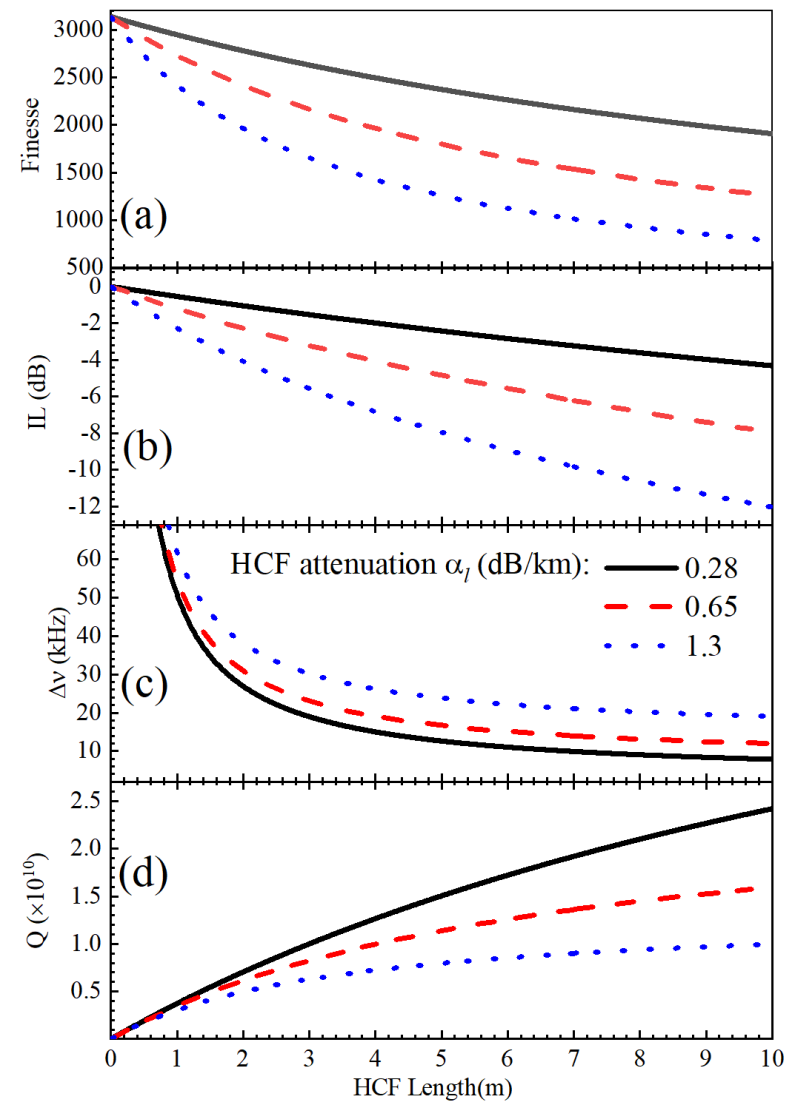

Fig. 4 Finesse (a), IL (b), $\Delta v$ (c), and Q (d) of HCF-FP versus HCF length for three values of the HCF loss (black solid, $0.28 \mathrm{~dB} / \mathrm{km}$; red dash, $0.65 \mathrm{~dB} / \mathrm{km}$; blue dot, $1.3 \mathrm{~dB} / \mathrm{km}$ ) with mirrors reflectivity of $99.9 \%$.

\section{FP ALIGNMENT AND CHARACTERIZATION}

\section{A. Characterization of finesse}

The optical scanning method [24] most-often used for FP characterization is rather impractical for FP alignment. This is because there are too many changing parameters during the alignment (FP peaks spectral position, insertion loss, polarization, etc.). Further, it requires a laser with a very narrow linewidth when dealing with high-finesse and long length (enabled by the use of optical fiber) FP fiber cavities. Thus, we used two different methods that proved to be more practical: the first one allows for fast coarse alignment, while the other one enables fast and fine FP alignment and its subsequent characterization. Both methods use incoherent unpolarized light from an erbium-doped fiber amplifier (EDFA). For the coarse alignment, we filtered a 10 -nm optical bandwidth from the EDFA that we launched into the FP and measured power at the FP output. Once maximum power was reached, we swapped the power meter with a fast photodiode and visualized and analyzed the output with a RF spectrum analyzer as shown in Fig. 5. The signal on the RF spectrum analyzer is given as:

$$
\begin{aligned}
P(f) & \propto\left|i_{n}(f)\right|^{2} \propto\left|\int t(v) t^{*}(f-v) d v\right|^{2} \\
& =\frac{1}{1+\left(\frac{2 F_{e}}{\pi}\right)^{2} \sin ^{2}\left(\frac{\pi f}{F S R}\right)},
\end{aligned},
$$

where $f$ denotes the RF frequency. $i_{n}(f)$ is the Fourier transform of the PD's photo-current signal which is proportional to the convolution of the transmission coefficient $t(v)$ and its conjugate. When deriving Eq. (8), we assumed that the optical spectrum of the incoherent source is flat which is reasonable over a limited bandwidth.

Although Eq. (8) has the same form as the optical transmission spectrum of a FP [24], it must be noted that $f$ is the $\mathrm{RF}$ frequency and $F_{\mathrm{e}}$ is

$$
F_{e}=\frac{\pi R_{0}}{1-R_{0}^{2}} \approx \frac{\pi \sqrt{R_{0}}}{2\left(1-R_{0}\right)}=\frac{F}{2}
$$

where $R_{0}$ is $R \cdot \mathrm{A}$ of the measured HCF-FPs. For a high-finesse FP in which $\mathrm{R} \sim 1$ like in our case, we have derived that $F \sim 2 F_{\mathrm{e}}$ based on the first order Taylor series approximation. Thus, the FP's RF spectrum has twice as broad peaks compared to the optical spectrum. In our experiment, data from the RF spectrum analyzer were acquired with a computer, where they were fitted with Eq. (8), enabling real-time evaluation of the $F S R$ and $F$ and thus also active FP alignment.

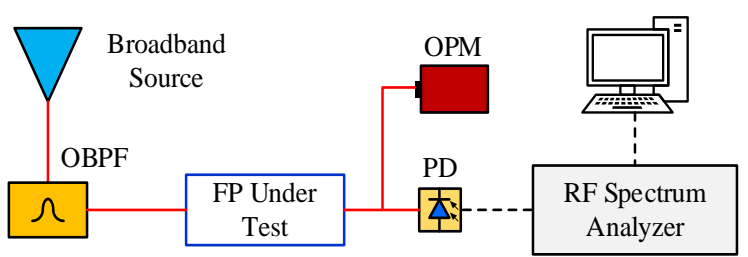

Fig. 5 FP characterization set-up, used also for active alignment. The broadband light source: Two cascaded Erbium-doped fiber amplifiers (EDFAs) passed through a 10-nm optical band-pass filter (OBPF). PD: photodetector. OPM: optical power meter.

\section{B. Evaluation of IL}

Besides $F$, the set-up in Fig. 5 also allows for evaluation of the $I L$. The total power loss (measured as the total power ratio after and before the FP) is proportional to the FP filtering characteristics (given by Eq. (8)) and IL. To enable straightforward experimental evaluation, we derived the following equation, which only requires measurement of the difference of the total optical power with and without the two mirrors in the set-up in Fig. 1 ( $\left.P_{d i f f}\right)$, and $F$ and FSR obtained in the previous sub-section:

$$
\mathrm{IL}=P_{\text {diff }}-10 \cdot \log _{10}\left(\int_{0}^{F S R} \frac{T(v)}{t_{\text {resonance }}} d v\right) .
$$




\section{RESULTS AND DISCUSSION}

We built five different HCF-FPs with lengths of $0.65,1.10$, $2.50,5.03$, and $9.25 \mathrm{~m}$.

\section{A. Measured data}

For illustrative purposes, we show the measured RF spectra and their fits (using Eq. (8)) only for the shortest and longestlength HCF-FPs, Fig. 6 . The small spikes at the bottom of the RF power spectra are artefacts originating from the EDFA electronics and were observed in all our measurements. The fits (shown in insets) were performed on the first RF peak (RF frequency corresponding to the FP's FSR).

The measured F, FSR, and $P_{\text {diff }}$ for all samples are summarized in Table 1.
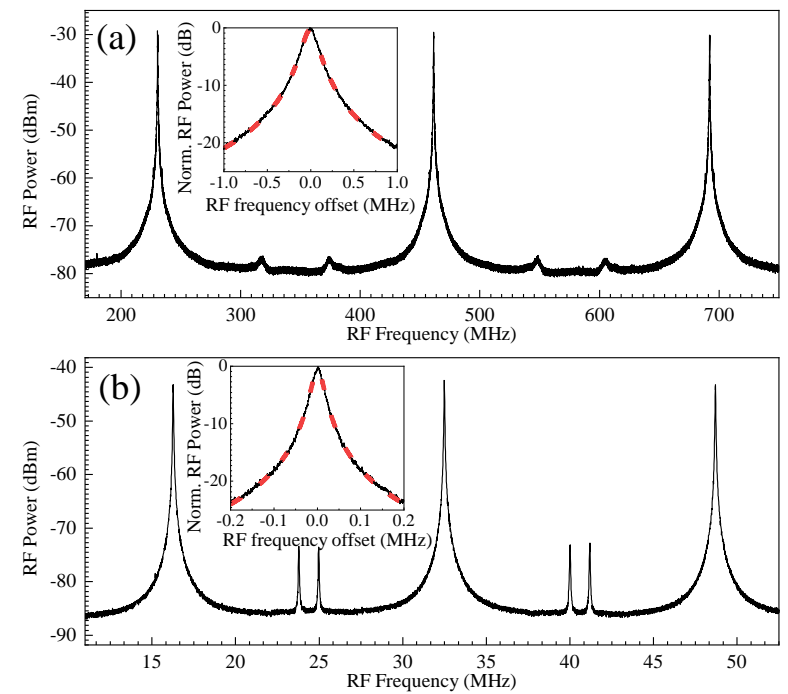

Fig. 6 RF spectra measured at HCF-FPs outputs with the (a) shortest $(0.65 \mathrm{~m})$ and (b) longest $(9.25 \mathrm{~m}) \mathrm{HCF}$ length. The inset shows the normalized spectrum of the first RF peak (black solid: measured; red dashed: fit using Eq. (8)). RF spectrum analyzer settings: (a) resolution bandwidth (RBW): $39 \mathrm{kHz}$, video bandwidth (VBW): $39 \mathrm{~Hz}$; (b) RBW: $10 \mathrm{kHz}$, VBW: $5 \mathrm{~Hz}$.

Table 1 Measured F and $P_{\text {diff }}$.

\begin{tabular}{|l|l|l|l}
\hline $\begin{array}{l}\text { HCF-FP } \\
\text { Length, } \mathrm{m}\end{array}$ & FSR, MHz & Finesse & $\mathrm{P}_{\text {diff }}, \mathrm{dB}$ \\
\hline 0.65 & 230.7 & 2430 & -38.0 \\
\hline 1.10 & 136.2 & 2245 & -38.0 \\
\hline 2.52 & 59.6 & 2003 & -39.5 \\
\hline 5.03 & 29.8 & 1600 & -39.7 \\
\hline 9.25 & 16.2 & 1212 & -41.3 \\
\hline
\end{tabular}

\section{B. F and IL}

$F$ (Table 1) and $I L$ (Eq. (10)) for all five HCF-FPs are shown in Fig. 7 (black, square dots). Both parameters degrade as the fiber length increases due to the signal attenuation in the fiber, as we have predicted (Fig. 4).

By re-writing Eq. (2):

$$
F=\frac{\pi \sqrt{R \cdot 10^{-\alpha_{L} L / 10}}}{1-R \cdot 10^{-\alpha_{L} L / 10}}
$$

and considering all HCF-FP samples have identical effective reflectivity $R$, we can fit the measured data shown in Fig. 7 (a), finding the effective reflectivity $R$ and HCF attenuation $\alpha_{l}$ (in $\mathrm{dB} / \mathrm{km}$ ) as fitting parameters. For our data, we found $\alpha_{l}=0.65$ $\mathrm{dB} / \mathrm{km}$ and $\mathrm{R}=99.88 \%$. The loss value agrees well with that measured with the cut-back measurement [30]. The effective reflectivity is consistent with the mirror specification (reflectivity >99.8\%) and with our mirror transmission measurement that put the upper bound of the mirror reflectivity to $99.94 \%$. This analysis thus reliably provides parameters relevant to the HCF $\left(\alpha_{l}\right)$ and we elaborate on this later in the Discussion section. It also provides key parameters necessary for accurate estimation of HCF-FP performance, as we show below.

The calculated $F$ using Eq. (11) and the fitted $R$ and $\alpha_{l}$ is plotted in Fig. 7 (a) (green solid line), showing excellent agreement with the measured $F$ data. Further, we calculated $I L$ using Eq. (4) with the fitted $\mathrm{R}, \alpha_{l}$, and measured mirror transmission $t_{1,2}$ (Fig. 7 (b), red solid line) which again shows excellent agreement with the measured $I L$ data.

The remarkable agreement between both fits and the measured data confirms the validity of our earlier assumption that $\mathrm{R}$ is identical for all HCF-FP samples, showing high repeatability of our $\mathrm{HCF}$ cleaving and mirror alignment.

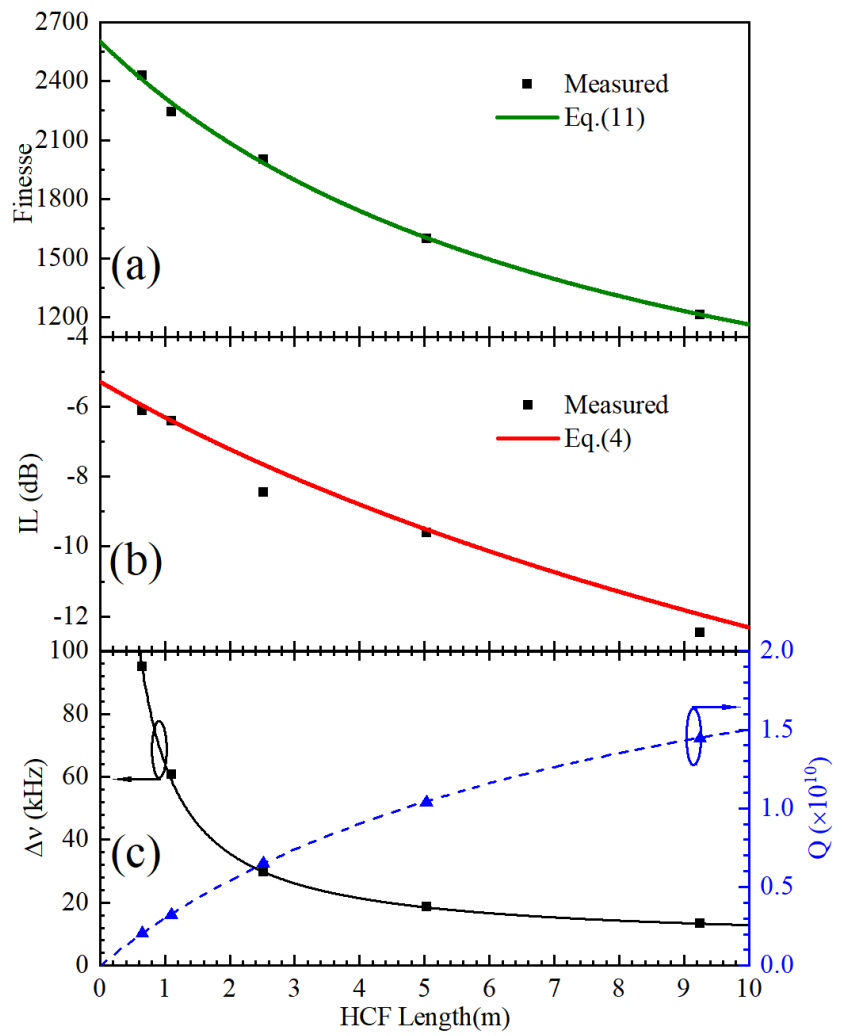

Fig. 7 (a) Finesse (measured, black squares); fitted with Eq. (11) (green solid line); (b) IL of HCF-FPs (measured, black squares) and processed with Eq. (4) (red solid line); (c) corresponding FWHM of transmission peaks $\Delta v$ (solid black) and Q (dashed blue) for different HCF lengths.

We also calculated FP transmission peaks FWHM $(\Delta v)$ and resonator quality factor $Q$ using Eqs. (3), (5) and (6), Fig. 7(c). For the longest FP $(9.25 \mathrm{~m})$, we achieved $\Delta v$ of $13 \mathrm{kHz}$ and Q over 14 billion. This corresponds to an effective light 
propagation distance inside the HCF-FP as long as $11 \mathrm{~km}$.

\section{Broadband characteristics}

Fig. 8 (a) shows transmission (measured output normalized to the input) of the 9.25-m HCF-FP measured with an Optical Spectrum Analyzer (OSA) with an optical resolution of $0.02 \mathrm{~nm}$. This resolution is too low to see the individual resonant transmission peaks but represents the transmission loss of the HCF-FP averaged over $0.02 \mathrm{~nm}$. This transmission loss changes by as much as $1.3 \mathrm{~dB}$ over the measured $5 \mathrm{~nm}$ bandwidth, which we believe is acceptable for most applications. However, it is worth analyzing its origin, which may suggest strategies as to how to reduce it.

To gain understanding, we first repeated the measurement without the two mirrors, Fig. 8 (a). Even without mirrors, we see a power variation. We performed a Fourier transform, which shows a peak around $2.6 \mathrm{ps} / \mathrm{m}$, which corresponds to the group delay between the NANF fundamental and $\mathrm{LP}_{11}$ mode, which is guided with significantly higher loss than the fundamental mode [30].
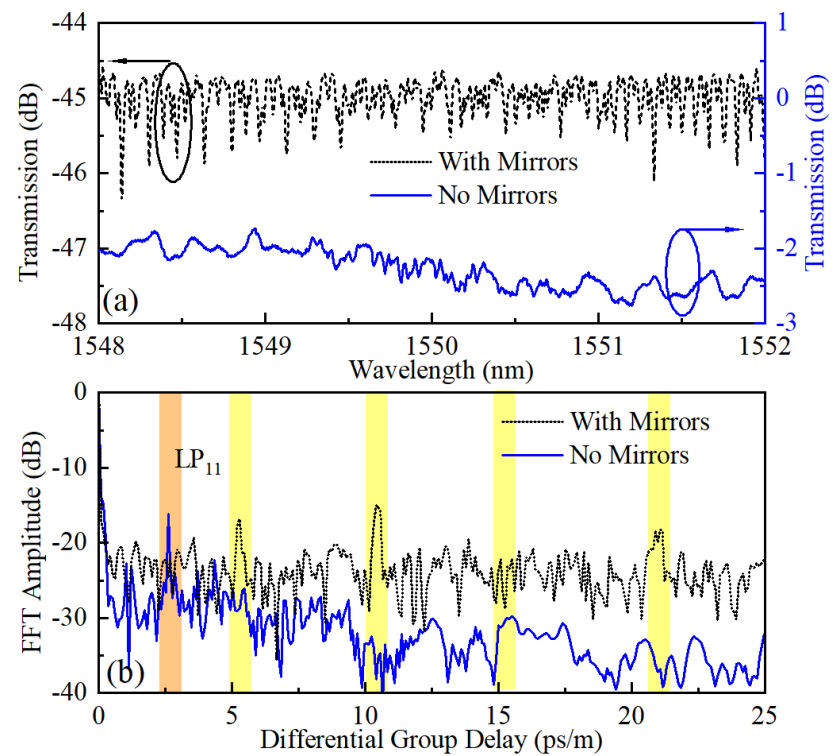

Fig. 8 (a) Transmission spectrum of the $9.25 \mathrm{~m}$ HCF-FP (dashed black line) and through HCF only (by removing the two mirrors, blue solid line) measured by OSA with $0.02 \mathrm{~nm}$ resolution. (b) Fourier transform of (a). Orange line shows expected position of the $\mathrm{LP}_{11}$ mode. Yellow lines show its double and its two harmonics.

Subsequently, we performed a Fourier transform of the HCF-FP transmission spectrum, Fig. 8 (b). We see the first peak corresponds to a double delay of the $\mathrm{LP}_{11}$ mode with another two peaks at the harmonics. We speculate these peaks are due to light remaining in the $\mathrm{LP}_{11}$ mode after successive round-trip(s) inside the HCF which then interferes with light in the fundamental mode, suggesting there is some level of coupling from the fundamental mode into the $\mathrm{LP}_{11}$ mode at the HCF-mirror interface. Due to the different symmetry of the $\mathrm{LP}_{11}$ mode and the fundamental mode, coupling between them requires some sort of asymmetry like an angle-cleave at the HCF or the mirror not being perfectly perpendicular to the reflecting beam.

\section{Discussion}

Our analysis of insertion loss suggested the effective mirror reflectivity to be $99.88 \%$. We measured the mirrors' transmission to be at least $0.055 \%$. This means we are losing $100-99.88-0.055=0.065 \%$. This loss is the sum of the mirrors internal loss and the coupling efficiency $\eta$. We do not know the internal loss, but can conclude that $\eta>99.935 \%$, which corresponds to a coupling loss of less than $0.0028 \mathrm{~dB}$. We calculated that such a coupling loss on its own limits the maximum achievable $F$ (for loss-less HCF and loss-less mirror) to 5000. To achieve it, however, would require mirrors with a reflectivity exceeding $>99.94 \%$. Indeed, values beyond 5000 could be achievable with better quality mirrors with lower internal loss than we have used. Experimentally, here we achieved an $F$ of up to 2400 and up to 3000 in an earlier report [29] in which we used mirrors with a slightly higher reflectivity than currently used. We have not used the same mirrors here, as they did not have an anti-reflective coating on the non-reflective side, making the alignment difficult and the performance not as broadband as demonstrated here. As we have demonstrated in Section IIIb, a HCF-FP can be used to measure the attenuation of the HCF, even when using a relatively short length of the fiber (10 $\mathrm{m}$ in our experiment). As loss reduction in HCFs continue, with very low loss (e.g., sub-0.3 dB [19]), now achieved, increasingly long lengths $(>1 \mathrm{~km})$ are needed to characterize the fiber attenuation accurately. Future lower-loss HCF will require even longer fiber samples. Our HCF-FP method needs significantly shorter fiber (10s of meters) and allows for attenuation characterization of different fiber segments (e.g., the beginning and the end of the draw), providing more data to feed back in to fiber manufacturing, as loss may vary along the fiber length [21]. Additionally, long length fiber is coiled on a drum and the measured loss may be influenced by this, e.g., micro bending loss [30].

\section{CONCLUSION}

Firstly, we analyzed theoretically how mirror tilt, distance between the $\mathrm{HCF}$ and the mirror, HCF cleave angle, and attenuation of state-of-the-art HCFs influence the performance of HCF-FPs. Following this, we constructed several HCF-FPs with HCF lengths between $0.65 \mathrm{~m}$ and $9.25 \mathrm{~m}$ and characterized them in terms of insertion loss, and finesse. Comparison of the experimental results with our theoretical analysis enabled us to estimate the key parameter that (together with the HCF attenuation) set the HCF-FP performance limits. We concluded that the coupling loss, defined in terms of the light lost when the HCF output is coupled back into the HCF via the mirror, is of critical importance. In our work the coupling loss was better than $0.0028 \mathrm{~dB}$ (corresponding to $99.94 \%$ of the light being coupled back into the HCF). We speculate this extremely low coupling loss number is achieved mainly thanks to the HCF cleave quality. This result suggests that similar performance could be achieved in a practical, alignment-free, all-fiber HCFFPs such as shown in [16], since all the factors limiting the finesse here and in [16] are identical.

The achieved low coupling loss would allow a finesse of over 
5000 provided mirrors with a high enough reflectivity were used. Experimentally, we achieved a finesse of up to 2430 with a HCF length of $0.65 \mathrm{~m}$ and a finesse of 1212 with a HCF length of $9.25 \mathrm{~m}$, limited by the above-mentioned coupling efficiency for short HCF-FP and HCF attenuation $(0.65 \mathrm{~dB} / \mathrm{km}$ used here) for the long HCF-FP. In terms of the transmission peak $\Delta v$ and $Q$ factor, we have achieved experimental values as low as 13 $\mathrm{kHz}$ for $\Delta v$ and as high as $1.45 \times 10^{10}$ for $Q$. This corresponds to an effective light propagation distance as long as $11 \mathrm{~km}$ inside the HCF-FP.

To achieve similar values in free-space-based FPs that are limited in their length, a finesse in excess of 110000 would be needed (considering a routinely-used FP length of $10 \mathrm{~cm}$ ), making the device extremely alignment sensitive and requiring mirrors with extremely high reflectivity/quality.

The achieved finesse of 2430 means that the power inside the cavity is 1550 times stronger than at the input, potentially enabling efficient (nonlinear) interaction with gases inside the HCF. This is of interest in a number of areas, e.g., in lasers, Raman sensing, or wavelength conversion and high-harmonic generation.

Finally, we demonstrated using a HCF-FP for the characterization of HCF loss using short fiber pieces, which is of interest for measuring loss in un-spooled samples (e.g., to eliminate any spooling contribution to loss) or for the characterization of low-attenuation HCFs.

\section{ACKNOWLEDGMENT}

The data in this paper is accessible through the University of Southampton research repository (DOI: 10.5258/SOTON/D1800).

\section{REFERENCES}

[1] A. V. V. Nampoothiri et al., "Hollow-core Optical Fiber Gas Lasers (HOFGLAS): a review [Invited]," Opt. Mater. Express, vol. 2, no. 7, pp. 948-961, 2012.

[2] S. Häfner et al., " $8 \times 10^{\wedge}-17$ fractional laser frequency instability with a long room-temperature cavity," Opt. Lett., vol. 40, no. 9, pp. 2112-2115, May 2015.

[3] T. Kessler et al., "A sub-40-mHz-linewidth laser based on a silicon single-crystal optical cavity," Nat. Photonics, vol. 6, no. 10, pp. 687692, Oct. 2012.

[4] G. Gagliardi, M. Salza, S. Avino, P. Ferraro, and P. De Natale, "Probing the Ultimate Limit of Fiber-Optic Strain Sensing," Science (80-. )., vol. 330, no. 6007, pp. 1081-1084, Nov. 2010.

[5] J. Chen, Q. Liu, and Z. He, "Time-domain multiplexed high resolution fiber optics strain sensor system based on temporal response of fiber Fabry-Perot interferometers," Opt. Express, vol. 25, no. 18, pp. 21914-21925, 2017.

[6] N. M. R. Hoque and L. Duan, "Ultrahigh-Resolution Fiber-Optic Sensing Based on High-Finesse, Meter-Long Fiber Fabry-Perot Resonators," IEEE Photonics J., vol. 12, no. 3, pp. 1-9, Jun. 2020.

[7] Y. Cui et al., " $4.3 \mu \mathrm{m}$ fiber laser in CO 2 -filled hollow-core silica fibers," Optica, vol. 6, no. 8, pp. 951-954, Aug. 2019.

[8] F. B. A. Aghbolagh et al., "Mid IR hollow core fiber gas laser emitting at 4.6 нm," Opt. Lett., vol. 44, no. 2, pp. 383-386, Jan. 2019.

[9] M. Xu, F. Yu, M. R. A. Hassan, and J. C. Knight, "Continuous-Wave $3.1 \mu \mathrm{m}$ Gas Fiber Laser with $0.47 \mathrm{~W}$ Output Power," in Conference on Lasers and Electro-Optics, 2017, p. SF2K.4.

[10] M. R. Abu Hassan, F. Yu, W. J. Wadsworth, and J. C. Knight, "Cavity-based mid-IR fiber gas laser pumped by a diode laser," Optica, vol. 3, no. 3, pp. 218-221, 2016.
[11] F. Benabid, F. Couny, J. C. Knight, T. A. Birks, and P. S. J. Russell, "Compact, stable and efficient all-fibre gas cells using hollow-core photonic crystal fibres," Nature, vol. 434, no. 7032, pp. 488-491, 2005.

[12] J. C. Travers, W. Chang, J. Nold, N. Y. Joly, and P. St. J. Russell, "Ultrafast nonlinear optics in gas-filled hollow-core photonic crystal fibers [Invited]," J. Opt. Soc. Am. B, vol. 28, no. 12, pp. A11-A26, 2011.

[13] F. Yang, F. Gyger, and L. Thévenaz, "Intense Brillouin amplification in gas using hollow-core waveguides," Nat. Photonics, vol. 14, no. 11, pp. 700-708, 2020.

[14] D. Munzke, M. Böhm, and O. Reich, "Gaseous oxygen detection using hollow-core fiber-based linear cavity ring-down spectroscopy," J. Light. Technol., vol. 33, no. 12, pp. 2524-2529, 2015.

[15] R. Slavík et al., "Ultralow thermal sensitivity of phase and propagation delay in hollow core optical fibres," Sci. Rep., vol. 5, no. 1, Art. no. 15447, Dec. 2015.

[16] M. Ding et al., "Long-Length and Thermally Stable High-Finesse Fabry-Perot Interferometers Made of Hollow Core Optical Fiber," $J$. Light. Technol., vol. 38, no. 8, pp. 2423-2427, 2020.

[17] W. Zhu et al., "Temperature insensitive fiber interferometry," Opt. Lett., vol. 44, no. 11,pp. 2768-2770, 2019.

[18] S. Wu, B. Siwicki, R. M. Carter, F. Biancalana, J. D. Shephard, and D. P. Hand, "Impact of nonlinear effects on transmission losses of hollow-core antiresonant negative curvature optical fiber," Appl. Opt., vol. 59, no. 16, pp. 4988-4996, 2020.

[19] G. T. Jasion et al., "Hollow Core NANF with $0.28 \mathrm{~dB} / \mathrm{km}$ Attenuation in the C and L Bands," 2020 Opt. Fiber Commun. Conf. Exhib. OFC 2020 - Proc., vol. 1, no. c, pp. 4-6, 2020.

[20] Y. Tamura et al., "The First 0.14-dB/km Loss Optical Fiber and its Impact on Submarine Transmission," J. Light. Technol., vol. 36, no. 1, pp. 44-49, Jan. 2018.

[21] H. Sakr et al., "Hollow core optical fibres with comparable attenuation to silica fibres between 600 and $1100 \mathrm{~nm}$," Nat. Commun., vol. 11, no. 1, Art. no. 6030, Dec. 2020.

[22] S. fei Gao, Y. ying Wang, W. Ding, Y. feng Hong, and P. Wang, "Conquering the Rayleigh Scattering Limit of Silica Glass Fiber at Visible Wavelengths with a Hollow-Core Fiber Approach," Laser Photonics Rev., vol. 14, no. 1, Art. no. 1900241, 2020.

[23] F. Poletti, "Nested antiresonant nodeless hollow core fiber," Opt. Express, vol. 22, no. 20, pp. 23807-23828, 2014.

[24] J. Flannery, R. Al Maruf, T. Yoon, and M. Bajcsy, "Fabry-Pérot Cavity Formed with Dielectric Metasurfaces in a Hollow-Core Fiber," ACS Photonics, vol. 5, no. 2, pp. 337-341, Feb. 2018.

[25] Y. Tan et al., "Hollow-Core Fiber-Based High Finesse Resonating Cavity for High Sensitivity Gas Detection," J. Light. Technol., vol. 35, no. 14, pp. 2887-2893, 2017.

[26] M. D. Wheeler, S. M. Newman, A. J. Orr-Ewing, and M. N. R. Ashfold, "Cavity ring-down spectroscopy," J. Chem. Soc. - Faraday Trans., vol. 94, no. 3, pp. 337-351, Jan. 1998.

[27] Y. Tan, W. Jin, F. Yang, Y. Jiang, and H. L. Ho, "Cavity-Enhanced Photothermal Gas Detection with a Hollow Fiber Fabry-Perot Absorption Cell," J. Light. Technol., vol. 37, no. 17, pp. 4222-4228, 2019.

[28] O. Volodarsky, Y. Hazan, and A. Rosenthal, "Ultrasound detection via low-noise pulse interferometry using a free-space Fabry-Pérot," Opt. Express, vol. 26, no. 17, pp. 22405-22418, 2018.

[29] M. Ding, E. R. N. Fokoua, T. D. Bradley, F. Poletti, D. J. Richardson, and R. Slavik, "Hollow core fiber Fabry-Perot interferometers with finesse over 3000," in 2020 Conference on Lasers and Electro-Optics (CLEO), 2020, Art. no. 2F2P.

[30] T. D. Bradley et al., "Antiresonant Hollow Core Fibre with 0.65 $\mathrm{dB} / \mathrm{km}$ Attenuation in the $\mathrm{C}$ and $\mathrm{L}$ Telecommunication Bands," in 2019 European Conference on Optical Communication (ECOC), 2019, Art. no. PDP3.1.

[31] Y. Amnon and P. Yeh, Photonics: optical electronics in modern communications. New York: Oxford university press, 2007.

[32] T. D. Bradley, J. R. Hayes, Y. Chen, G. T. Jasion, S. R. Sandoghchi, and R. Slavik, "Record Low-Loss 1 . 3dB / km Data Transmitting Antiresonant Hollow Core Fibre," 2018 Eur. Conf. Opt. Commun., no. 1, pp. 1-3, 2018. 